\title{
Efficacy and Safety of Modified Bushen Yiqi Formulas(MBYF) as Add-On To Formoterol and Budesonide in The Management of COPD: Study Protocol for A Multi-Center, Double-Blinded, Placebo-Controlled, Parallel-Group, Randomized Clinical Trial
}

\section{Qing Kong}

Huashan Hospital Fudan University Department of Integrative Medicine

\section{Yuxue Cao}

Huashan Hospital Fudan University Institute of Integrative Medicine: Huashan Hospital Fudan University Department of Integrative Medicine

\section{Zhen Gao}

Huashan Hospital Fudan University Institute of Integrative Medicine: Huashan Hospital Fudan University Department of Integrative Medicine

\section{Jing Sun}

Huashan Hospital Fudan University Institute of Integrative Medicine: Huashan Hospital Fudan University Department of Integrative Medicine

\section{Hongying Zhang}

Huashan Hospital Fudan University Institute of Integrative Medicine: Huashan Hospital Fudan University Department of Integrative Medicine

\section{Yijie Du}

Huashan Hospital Fudan University Institute of Integrative Medicine: Huashan Hospital Fudan University Department of Integrative Medicine

\section{Yubao LV}

Huashan Hospital Fudan University Institute of Integrative Medicine: Huashan Hospital Fudan University Department of Integrative Medicine

\section{Sihan Zhou}

Huashan Hospital Fudan University Institute of Integrative Medicine: Huashan Hospital Fudan University Department of Integrative Medicine

\section{Zihui Tang}

Huashan Hospital Fudan University Institute of Integrative Medicine: Huashan Hospital Fudan University Department of Integrative Medicine 
Huashan Hospital Fudan University Institute of Integrative Medicine: Huashan Hospital Fudan University Department of Integrative Medicine

Jingcheng Dong ( $\sim$ jingcheng_dong@yeah.net )

Huashan Hospital Fudan University Institute of Integrative Medicine: Huashan Hospital Fudan University Department of Integrative Medicine

\section{Study protocol}

Keywords: Randomized clinical trial, Design and method, COPD, Traditional Chinese Medicine

Posted Date: June 28th, 2021

DOl: https://doi.org/10.21203/rs.3.rs-434972/v1

License: (c) (1) This work is licensed under a Creative Commons Attribution 4.0 International License. Read Full License 


\section{Abstract}

Introduction Inhaled glucocorticoid Corticosteroids(ICS), long-acting $\beta 2$-adrenoceptor agonists(LABA)and other drugs have limited therapeutic effects on COPD with significant individual differences. Traditional Chinese Medicine (TCM) modified Bushen Yiqi formulas $\square$ MBYF $\square$ demonstrates advantages in COPD management in China. This study aims to confirm the related genes affecting the therapeutic effect in the treatment of COPD and evaluate the efficacy and safety of MBYF as an add-on to budesonide/ formoterol on COPD patients.

Methods and analysis In this multicenter, randomized, double-blinded, placebo-controlled, parallel-group study, eligible patients with COPD will randomly receive 360-day placebo or MBYF as an add-on to budesonide/ formoterol in 1:1 ratio, and followed-up bimonthly. The primary outcomes are frequency, times and severity of acute exacerbation of COPD (AECOPD), COPD assessment test (CAT) score, pulmonary function tests(PFTs). The secondary outcomes include the modified medical research council

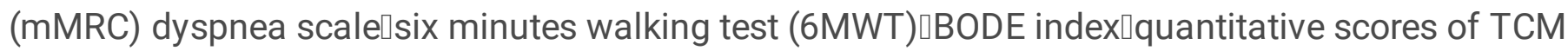
syndrome inflammation indexes and hypothalamic pituitary adrenaline (HPA) axis function. We also test the genotype to find the relationship between drugs and efficacy. All the data will be recorded in case report forms(CRFs) and analyzed by SPSS V.20.0.

Trial registration number ChiCTR1900026124, Prospective registration.

Protocol version 2019-09-15

\section{Introduction}

Chronic Obstructive Pulmonary Disease (COPD) will become the third leading cause of death worldwide in 2030 and nearly $90 \%$ of COPD deaths occur in low-and middle-income countries(1) In China, $13.7 \%$ of the population over forty years old suffer from COPD, creating a large socio-economic burden(2-4). COPD is characterized by progressive airflow limitation caused by chronic inflammation of the airway and lung parenchyma(5). Patients with COPD are inflicted by lasting respiratory symptoms and systemic manifestations, such as cardiovascular disease, osteoporosis, muscle weakness, depression, and lung cancer will lead to disability and impaired quality of life $(6,7)$. However, current COPD therapies like inhaled corticosteroids (ICS) and $\beta 2$-adrenoceptor agonists( $L A B A)$ are merely symptomatic but not effectively relieve the oxidative stress and improve the trend of declining pulmonary function(8-10). And long-term ICS can be ineffective or even associated with the undesired side, including osteoporosis, peptic ulcer bleeding, hypertension, etc $(11,12)$. Moreover, drug response varies greatly among individuals, so better methods are needed to identify who can benefit instead of taking risks. Therefore, under the existing medical conditions, the treatment of COPD solely relying on modern medicine has encountered a bottleneck, finding new drugs and methods for the intervention of COPD is necessary.

Traditional Chinese Medicine(TCM) has great advantages in relieving symptoms, improving the life quality of patients, enhance the efficacy and reduce the side effects of modern medicine like 
glucocorticoids in the long experience and evidence-based clinical practice(13-16). COPD is considered as an ageing-related disease following with a group of TCM syndromes of deficiency and stasis, which refers to the weakness of the lungs, spleen and kidney both in the stable period and onset period. Recently, real-world evidence-based studies have proved the dominant distribution of this syndrome called ("Fei_Shen_Qi_Xu" Zheng in Chinese) $(17,18)$. We have observed that Bushen Yiqi formulae(BYF), a herbal formula targeting for this syndrome according to TCM theory, demonstrates effective in ameliorating symptoms, reducing exacerbations, improving the pulmonary function in the treatment of COPD in clinical(19). BYF consist of three herbs as show in Table 1. As the clinical experience accumulate, we find that Modified BYF(MBYF), the BYF added with two additional two herbs, shows more effectives than BYF on COPD patients. MBYF consists of five herbs as shown in Table 1.

Furthermore, vivo and vitro experiments show that MBYF and its compounds can significantly adjust inflammation and immunology(20-23). Icariin in Yinyanghuo has anti-inflammatory effects, and upregulate the amount of $\mathrm{GRa}$ and promoted its nucleus translocation in inflammatory cell models and lung tissue of inflammation animal model $(24,25)$. Astragaloside in Hungqi reduces the protein expression of JAK3/STAT3/NF-B pathway in CS-induced COPD mice(26). Scutellaria baicalensis in Huang Qin exerts lung function protection, proinflammatory and anti-inflammatory cytokine regulation, anti-airway remodelling, and antioxidant role in long term CS-induced COPD model $(27,28)$. Paeonol in Chi Shao has anti-inflammatory and antioxidant functions against CS-induced lung inflammation in both vivo and vitro $(29,30)$.

Therefore, we propose a hypothesis that MBYF benefit COPD patients in exacerbation reduction, symptoms amelioration and pulmonary function improvement, etc. Therefore, we design a multicenter, double-blinded, randomized clinical trial to evaluate the efficacy and safety of MBYF as an add-on to budesonide/ formoterol on patients with COPD.

Besides, this study drew precision medical model into TCM intervention in COPD trial. Precision medicine pays attention to the depth characteristics of "disease" and the high precision of "medicine", which accurately to find the disease classification, finally realizes personalized expectant treatment for patients with specific purposes, improve the benefit of disease diagnosis $\square$ treatment and prevention. At present, the shreds of evidence for the precise treatment of asthma has been obtained. For example, the detection of the corticotropin-releasing hormone receptor 1 gene (CRHR1) can predict the short-term therapeutic effect of ICS $(31,32)$. FCER2 gene can predict long-term treatment of ICS $(33,34)$. ADRB2 gene can predict the efficacy of $\beta$-agonists and anticholinergic drugs. For Arg/Arg type, SABA or LABA should not be used, while for Gly/Gly type, SABA or LABA can significantly improve lung function. In contrast, patients with Arg/Arg type asthma can be treated with Tiotropium Bromide Powder for Inhalation: the response rate is as high as $60 \%(35)$. Glucocorticoid-induced transcript 1 gene (GLCCl1) variant (RS 37972C/T and RS $37973 \mathrm{~A} / \mathrm{G}$ ) can affect the effect of glucocorticoid therapy in patients(36-38). Do genes that influence the efficacy of ICS, and salmeterol for asthma also influence the efficacy of COPD? Therefore, this study also detects genes that influence the efficacy of ICS/LABA and other drugs in the treatment of COPD, to provide evidence to clinical use. 


\section{Method And Analysis}

\section{Study design}

This is a multicentre, randomized, double-blinded, placebo-controlled, parallel-group clinical trial. We will rigorously follow the latest Consolidated Standards of Reporting Trials (CONSORT 2017) for Chinese herbal Medicines recommendations(39), and compile with the principles of the Declaration of Helsinki and Good Clinical Practice guidelines. Standard Protocol Items: Recommendations for Interventional Trials and 2013 statement for herbal interventions(40). Totally 300 participants diagnosed COPD will be enrolled in the trial, which consists of a 7-day screening period and a 360-day treatment period with every 60-day follow-up. In addition to routine medications, patients will be given $10.24 \mathrm{~g} \mathrm{MBYF}$ or placebo in granule form two times per day for 360 days. The participant's flowchart is briefly illustrated in Figure 1.

\section{Participant recruitment}

Consecutive patients undergoing pulmonary function tests(PFTs) for a clinical indication will be evaluated for the entry into the study. Inpatients and outpatients at the ten participating hospitals will be screened according to the inclusion and exclusion criteria by two experienced respiratory physicians separately. Table 2 shows the cooperative hospital recruiting patients. The participants will be enrolled in the trial only when confirmed by two respiratory physicians at the same time. Also, recruitment advertisements of the study will be posted on webpages and notice boards in ten participating hospitals and resident communities. It includes a brief description of the subjects needed, the medicines, medical examinations and the ways to participate in this study. For those people who are ineligible or decline to participate, we will record the basic demographic information and reasons for non-participation. The first volunteer has been recruited on January 14, 2020 in Xinjiang. Due to COVID-19, we expect to complete the recruitment process around December 2020 and report the results as soon as possible.

\section{Diagnostic criteria of COPD}

The diagnosis of COPD will refer to the GOLD science committee report 2019. According to the comprehensive analysis of clinical manifestations, risk factor exposure history, signs and laboratory examinations and other data. Consider the main symptoms of COPD as chronic cough, sputum and/or dyspnea and risk factor exposure history. The incomplete reversible airflow limitation is a necessary condition for the diagnosis of COPD. The gold standard for COPD is FEV1/FVC $<70 \%$ after using bronchodilators. Anyone with a history of smoking and/or exposure to environmental occupational pollution and/or cough, sputum or difficulty breathing, will be screened by PFTs. Chest x-ray examination helps determine the degree of the hyperinflation of the lungs. Exclude bronchial asthma, bronchiectasis, congestive heart and other lung diseases such as heart failure and tuberculosis.

\section{Diagnostic criteria of TCM syndrome}

TCM syndrome of 'Fei_Shen_Qi_Xu' will refer to "Clinic terminology of traditional Chinese medical diagnosis and treatment-Syndromes, GB/T 16751.2-1997". Table 3 shows the diagnostic criteria of 
'Fei_Shen_Qi_Xu'. If patients have two of the primary symptoms and two of the secondary symptoms, TCM syndrome of 'Fei_Shen_Qi_Xu' is determined.

\section{Inclusion criteria}

(1) Patients clinically diagnosed as COPD with airflow limitation: a post-bronchodilator fixed ratio FEV $1 /$ FVC $<0.7,30 \% \leq \mathrm{FEV} 1<80 \%$ predicted.

(2) Patients without respiratory tract infection and acute exacerbation of COPD (AECOPD) in the past four weeks;

(3) Patients with TCM syndrome of 'Fei_Shen_Qi_Xu';

(4) Patients with severe exacerbation history: $\geq 2$ or $\geq 1$ leading to hospital admission in the past year;

(5) An age limit ranged from 40 to 85 years;

(6) Provision of written informed consent by participants or surrogates voluntarily.

\section{Exclusion criteria}

(1) Airflow obstruction caused by asthma and other non-COPD;

(2) Patients with lung transplantation or pneumonectomy;

(3) Patients needing long-term oxygen therapy (daily oxygen inhalation time $\geq 12$ hours);

(4) Patients with a history of chronic alcoholism or drug abuse;

(5) Patients with the standard treatment of oral glucocorticoid at present;

(6) Patients with the treatment of pulmonary rehabilitation;

(7) Patients accompanied by serious diseases of heart, liver and kidney;

(8) Patients with a malignant tumour, leukaemia, aplastic anaemia, myelodysplastic syndrome, thrombocytopenia, multiple myeloma and other haematological diseases;

(9) Patients who are allergic or intolerant to the study of drugs;

(10) Participate in other clinical trials in the past four weeks;

(11) Pregnant women or breastfeeding women;

(12) Other.

\section{Removal, dropout and termination criteria}

Participants not taken medication during the treatment period will be removed. Participants can voluntarily drop out at any time during the trial. Eligible subjects failing to complete the observation period will be considered as dropout cases regardless of the time and reason. Reasons for dropout will be recorded in the case report forms(CRFs), and the last data recorded for these participants will be included in the data analysis. The trial will be suspended if serious adverse events (SAEs) relevant to the MBYF occur, or the participant decides to join in another clinical research project in terms of COPD, or demonstrates hypersensitivity towards MBYF, such as abnormal thirsty, stomach ache and diarrhoea, or suffers from the acute life-threatening disease, or with a combination of drugs, especially the drugs that affect the efficacy and safety of the MBYF. 
The whole research will be terminated in the following circumstances: (1) masking of the randomization fails; (2) unblinding rate exceeds $20 \%$ of the sample size; (3) assessments of all follow-up are completed.

\section{Randomization and blinding}

According to the random number sequence generated by SAS, the patients will be randomly divided into either the MBYF group or the placebo group in a 1:1 ratio, using SAS to achieve computerised randomisation in blocks of 4 , stratified by center. We set the blind code in the case for remaining blind when the patients have adverse effects. The random code and blind code will be conducted by a "third party" independent of the study using opaque envelopes. The envelopes will be sealed and shuffled and the assignment records will not be disclosed until the end of the study. To ensure the implementation of the blinding method, MBYF and MBYF placebo will be made in the same shapes, smells and tastes. Trial participants, care providers including attending physician and nurse, outcome assessors including PI and sub-PI, and data analysts will be blinded after the assignment of interventions.

\section{Procedure for unblinding}

If there is sevea re adverse event, which impedes the progress of the trial and the selection of the treatment measures, urgent unblinding can be carried out. During the process, all the researcher, sub-PI, and clinical supervisors should take part. The local administrative unit should be informed within 24h. The reason, time, and place of unblinding should be recorded in detail and all the records should be signed off.

\section{Interventions}

Budesonide formoterol powder inhaler $(160 \mu \mathrm{g} / 4.5 \mu \mathrm{g}$, once in the morning and evening, 2 inhalations each time) is provided as a conventional treatment to all participants according to the GOLD for COPD. MBYF or placebo( $10.24 \mathrm{~g} / \mathrm{bag}$, one bag at a time, two times per day, 360 days) is provided as adjunct medication randomly using an envelope. MBYF and placebo(contain 10\% MBYF) are identical in both taste $\square$ appearance and package and produced by Huarui Sanjiu pharmaceutical industry in Shenzhen, China. Granule production was certified to the standard certification of the TCM National Drug Regulatory Authority (41).

\section{Interventions modifications}

Modification or discontinuation of the intervention will be decided by the PIs in each center, according to the requests from participants, or when AECOPD occurs which indicates the need for ICU admission, or when unexpected adverse effects happen.

\section{Compliance}

MBYF are free as study drugs and will contribute to subjects bimonthly. All unused packs of drugs and empty bags will be returned to investigator bimonthly. Compliance will be calculated by counting drugs or 
empty bags: Compliance $\%$ of medication $=$ [actual dose $/($ specified daily dose $\times$ days) $] \times 100 \%$. Total medication consistency ranging from 80 to $120 \%$ will be eligible for the protocol analysis set. Parts of the laboratory tests will be performed on standard schedule freely, which aids in the monitoring of adherence.

\section{Concomitant care}

Any drugs or care are permitted when AECOPD occurs. Other ICS and bronchodilators are prohibited during a stable period. Herbal drugs not related to "Tonifying Shen" are allowed to use as disease needs.

\section{Data collection}

Items to be measured and the time window of data collection are shown in Table 4. After screening and completing baseline evaluations, participants will join in a Wechat group where we will provide free consultation by physicians. The participants and their family member will be informed that standardized treatment is beneficial to reduce COPD exacerbation, which will reduce medical expenses the benefits. Telephone calls will be conducted on communication monthly.

\section{Data management and monitoring}

All the information in the CRF table will be recorded in a specialized clinical experimental database. Details of data management procedures are similar to the other protocol which we have published(42) (https://www.ncbi.nlm.nih.gov/pubmed/32883322).

The data monitoring committee is composed of an investigator from Huashan hospital due to no sponsor. A data monitoring committee will audit the trial conduct via internet meeting monthly and site investigation semi-annually. An interim analysis will be conducted to evaluate the safety of MBYF when 30 subjects have been followed up at 180-days. Pls and the ethics committee have access to these results and will make the final decision to terminate the trial. For all adverse events, the time, duration, treatment measures and outcomes, the severity of the disease, and the association with the study drug will be evaluated and recorded. If an AE occurs, the doctor will immediately take emergency measures and report it to the Pls and the ethics committee within $24 \mathrm{~h}$.

\section{Background information}

Background information includes demographic data(gender, age, height, weight and so on) and general clinical data(medical history, course of the disease, treatment history, combined diseases, concomitant medications and so on), which will be recorded during the 1-week screening period. The participants' information and privacy will be strictly protected and forbidden to the public.

\section{Safety outcomes}

Safety will be assessed at 180 days and 360 days after randomization by electrocardiogram (ECG), chest X-ray or computed tomography (CT), laboratory examinations(blood and urine routine, liver and kidney 
function, glucose.) and adverse events(AEs). AEs will be recorded all the time during the treatment and observed until disappear.

\section{Primary outcomes}

(1)The frequency $\square$ time and severity of AECOPD

The frequency $\square$ time and severity of AECOPD will be collected at every 60 days after randomization. AECOPD is defined as the patient presents with two of the following symptoms (at least one primary symptom) which last at least 2 days. Primary symptoms: Dyspnea, profuse sputum, purulent sputum; secondary symptoms: Wheezing, pain in the throat, cough and the common cold, etc. The interval of AECOPD between two episodes is defined as at least a week. Time of AECOPD is defined as the duration from acute exacerbation to the apparent improvement of the symptoms (the patient's self-sensation). The severity of AECOPD is defined as three degrees. Mild: no need for clinic service; oral intake of hormones, antibiotics and oxygen therapy are necessary. Moderate: clinic service is necessary, and oral intake of hormones, antibiotics or oxygen therapy is required. Severe: hospitalization or emergency treatment is needed.

(2) COPD assessment test (CAT) score

CAT score will be used for evaluation of symptom at baseline $₫ 180$ days and 360 days after randomization. Please see the supplementary file.

(3) Pulmonary function tests(PFTs)

A post-bronchodilator forced expiratory volume in 1 second (FEV1) \% predicted 0 forced vital capacity (FVC) ratio (FEV1 /FVC) and blood gas analysis will be measured at baseline $₫ 360$ days after randomization. Before the test, the treatment drugs should be discontinued for more than 6 hours, violent activity should be discontinued for 2 hours, and patients should rest for 15 minutes.

\subsubsection{Secondary outcomes}

(1) Modified Medical Research Council (mMRC) dyspnea scale

mMRC will be used for evaluation of dyspnea at baseline $₫ 180$ days and 360 days after randomization. Please see the supplementary file.

(2) Six minutes walking test (6MWT)

6MWT will be used for evaluation of aerobic exercise capacity at baseline $₫ 180$ days and 360 days after randomization. The patient will be asked to walk quickly for 6 minutes and the distance will be recorded. Dyspnea scales, fatigue, respiratory rate, heart rate and oxygen saturation before and after walking are calculated. Borg scale will be used to evaluate the severity of dyspnea and fatigue. Please see the supplementary file. 
(3) BODE index

BODE will be used for prediction of the mortality rate at baseline $₫ 180$ days and 360 days after randomization. It includes the following aspects: $\triangle \mathrm{B}$ : body mass index (BMI) $\triangle \mathrm{D} \otimes \mathrm{D}$ egree of airflow

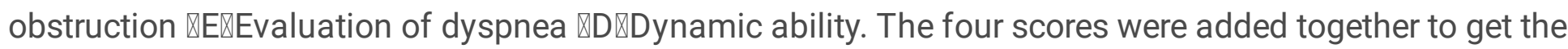
BODE score. Please see the supplementary file.

(4) Quantitative scores of TCM syndrome

Quantitative scores of TCM syndrome will be used for evaluation of the TCM syndrome of Fei_Shen_Qi_Xu at baseline $₫ 180$ days and 360 days after randomization. The treatment index $(n)=$ scores before the treatment-scores after the treatment $₫ /$ scores before the treatment $100 \%$. Please see the supplementary file.

(5) Inflammation indexes and hypothalamic pituitary adrenaline(HPA) axis function

Inflammation indexes and HPA axis function will be evaluated at baseline $\ 180$ days and 360 days after randomization. Draw $10 \mathrm{ml}$ of blood and separate the serum $(2-3 \mathrm{ml})$ which is sent to Huashan Hospital

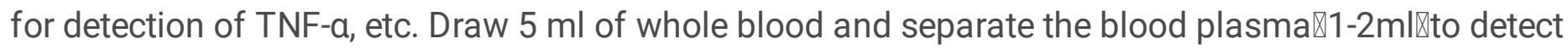
hydrocortisone.

\section{Other tests}

$5 \mathrm{ml}$ Blood will be drawn at baseline, and peripheral blood mononuclear cells will be isolated to detect CRHR1, FCER2, GLCCI1 and other genotypes. The relationship between drug efficacy and genotype will be evaluated.

\section{Sample size calculation}

According to the primary outcomes in the previous trial $(14,19)$, at the $5 \%$ significance level, a total of 118 patients per group will be required for a 2-group, the 1-sided calculation to achieve $95 \%$ power and the differences of $(10.30 \pm 6.31,12.95 \pm 5.99)$ in CAT mean score between the TCM treatment group and placebo group, as calculated using G-power 3.1 software. A loss of $15-20 \%$ to follow-up is predicted based on experience--this increase the sample size to 142 participants per group. Based on a 1:1 ratio, the final decision is 300 in total.

\section{Adverse events(AEs)}

AEs are defined as negative or unintended clinical manifestations following the treatment. Patients will be asked to report to the investigators any abnormal reactions occurring at any time during the trial. Besides, investigators will collect information about abnormal reactions every 60 days. All details include the time of occurrence, degree and duration of AEs, suspected causes and the effective measures and outcomes will be recorded on CRFs. Subjective discomfort, abnormalities of ECG, chest X-ray or CT and laboratory, should be taken seriously. Careful analysis and immediate measures will be taken to protect 
the safety of the subjects until the AEs disappeared. There is also a data safety monitoring board to oversee the trial.

\section{Quality control of data}

CRFs will be used for data collection. All investigators will receive pretrial training on patients screening, data filling, medication use, AEs reporting and other matters. A trial inspector will visit each hospital regularly to check the electronic database and ensure the protocol is strictly followed. The Pls will be in charge of data validation. and take measures to control the drop-out rate within $15 \%$.

\section{Planned analysis}

Baseline data and efficacy will be analyzed using the modified intention-to-treat (MITT) population, and the safety outcomes were analyzed using the safety analysis population (SAP). The Kolmogorov-Smirnov test will be used to determine whether or not continuous variables followed a normal distribution. Unnormal distributional variables will be log-transformed to approximately normal distributions before analysis. Continuous variables will be expressed by Mean \pm SD or the Median, categorical variables will be shown as counts and percentages. Differences among the two groups will be compared with the use of analysis of variance $\triangle A N O V A \otimes f$ for continuous variables and the $X^{2}$ test for categorical variables. Fisher's least significant difference test will be used to explore further and compare the mean of two groups. The analysis of covariance will be used for controlling potential confounding variables. Statistical package for social sciences for windows version 16.0 (SPSS, Chicago, IL, USA) will be used for analysis. Twosided tests will be used throughout, with the statistical significance level set at 0.05 , and $95 \%$ Cls will be used for interval estimation. The methods of analysis of each variable are summarized in Table 5.

\section{Ethics and dissemination}

This study has been approved by the Ethics Review Committee of Huashan Hospital affiliated to Fudan University, Shanghai, China (Provisional Trial No. (425) 2018), and registered at the Chinese Clinical Trial Registry http://www.chictr.org.cn/ at15 December 2019(ChiCTR1900026124). Written informed consent will be acquired from all patients before treatment.

\section{Patient and public involvement}

No patient or public was involved in the design of this study. The results will be disseminated to the public through academic conferences and peer-reviewed journals, and briefly sent to all participants through Wechat.

\section{Discussion}

In this multicenter, randomized, double-blinded, placebo-controlled clinical trial, we will clarify a pragmatic method to identify the efficacy and safety of MBYF as an adjunct to budesonide/formoterol to treat COPD. A simple, convenient, cheap and effective herbal formula based on TCM syndrome differences 
might strongly support and enrich the management of COPD. The level of HPA axis and pro/antiinflammation between MBYF and placebo groups might indicate that mechanism of TCM treatments on COPD or other ageing diseases. The exploration of the relationship between genotype and ICS/LABA/other drugs will provide powerful evidence and guideline to the COPD individual therapy. In all, the finding is potential to form a unique diagnosis and treatment scheme combining Traditional Chinese medicine and Modern medicine and promote its application.

However, there are some limitations to this protocol. First, we choose one of the specific TCM syndromes as the criteria. The result is hard to be extended to the whole patients with COPD exacerbation. Second, the bias of measurement and the subjectivity of the questionnaire assessment is inevitable, which may be exacerbated by the loss of some participant during follow-up. Third, the trial will be conducted in ten hospitals of China, and whether similar effects are available to other ethnic groups and regions remains uncertain.

\section{Trial Status}

Our trial has enrolled 30 volunteers from 14 January 2020 up to today. We have modified protocol according to the practice and standard protocol items $(40,43)$. The protocol version is $20190905-2$. The new protocol was reported to all sub-center PIs in a group meeting. Due to COVID-19, we expect to complete the recruitment process around November 2021 and report the results as soon as possible.

\section{Contributor}

$\mathrm{JD}$ and $\mathrm{BL}$ is the principal investigator of this study. QK and $\mathrm{YC}$ contributed equally to the article, who wrote and modified the manuscript. ZG, JS, and HZ conceptualized the study design. YD, YL and SZ participated in the recruitment of patients. ZT designed the method for statistic analysis. All authors read and approved the final manuscript.

\section{Declarations}

\section{Funding}

This study is supported by Shanghai three-year Action Plan for further speeding up the Development of Traditional Chinese Medicine (2018-2020) (No.ZY (2018-2020)-CCCX-4002] $ه$ Shanghai science and technology commission scientific research project (No.19401931400), Chinese medicine innovation project of Shanghai Health Committee $₫$ No.ZYKC201601023》, and National Natural Science Foundation of China(No.81501180)

\section{Competing interests}

None declared. 


\section{References}

1. The top 10. causes of death 2018 [Available from: https://www.who.int/zh/news-room/factsheets/detail/the-top-10-causes-of-death.

2. Burden of Chronic Obstructive Pulmonary Disease: World Health Organization; 2019 [Available from: https://www.who.int/respiratory/copd/burden/en/.

3. Wang C, Xu J, Yang L, Xu Y, Zhang X, Bai C, et al. Prevalence and risk factors of chronic obstructive pulmonary disease in China (the China Pulmonary Health CPH study): a national cross-sectional study. Lancet. 2018;391(10131):1706-17.

4. Zhou M, Wang H, Zeng X, Yin P, Zhu J, Chen W, et al. Mortality, morbidity, and risk factors in China and its provinces, 1990-2017: a systematic analysis for the Global Burden of Disease Study 2017. Lancet. 2019;394(10204):1145-58.

5. Rabe KF, Watz H. Chronic obstructive pulmonary disease. The Lancet. 2017;389(10082):1931-40.

6. Vanfleteren L, Spruit MA, Wouters EFM, Franssen FME. Management of chronic obstructive pulmonary disease beyond the lungs. Lancet Respir Med. 2016;4(11):911-24.

7. Barnes PJ, Celli BR. Systemic manifestations and comorbidities of COPD. Eur Respir J. 2009;33(5):1165-85.

8. Barnes PJ. Targeting cytokines to treat asthma and chronic obstructive pulmonary disease. Nat Rev Immunol. 2018;18(7):454-66.

9. Singh D, Agusti A, Anzueto A, Barnes PJ, Bourbeau J, Celli BR, et al. Global strategy for the diagnosis, management, and prevention of chronic obstructive lung disease: the GOLD science committee report 2019. The European respiratory journal. 2019.

10. Szafranski W, Cukier A, Ramirez A, Menga G, Sansores R, Nahabedian S, et al. Efficacy and safety of budesonide/formoterol in the management of chronic obstructive pulmonary disease. Eur Respir $\mathrm{J}$. 2003;21(1):74-81.

11. Agusti A, Fabbri LM, Singh D, Vestbo J, Celli B, Franssen FME, et al. Inhaled corticosteroids in COPD: friend or foe? The European respiratory journal. 2018;52(6).

12. Toogood JH. Side effects of inhaled corticosteroids. J Allergy Clin Immunol. 1998;102(5):705-13.

13. Haifeng W, Hailong Z, Jiansheng L, Xueqing Y, Suyun L, Bin L, et al. Effectiveness and safety of traditional Chinese medicine on stable chronic obstructive pulmonary disease: A systematic review and meta-analysis. Complement Ther Med. 2015;23(4):603-11.

14. Ma JF, Zheng JP, Zhong NS, Bai CX, Wang HY, Du J, et al. Effects of YuPingFeng granules on acute exacerbations of COPD: a randomized, placebo-controlled study. International Journal of Chronic Obstructive Pulmonary Disease. 2018;13:3107-14.

15. Jiansheng L, Haifeng W, Suyun L, Hailong Z, Xueqing Y, Xiaoyun Z, et al. Effect of sequential treatment with TCM syndrome differentiation on acute exacerbation of chronic obstructive pulmonary disease and AECOPD risk window. Complement Ther Med. 2016;29:109-15. 
16. Wang Y, Xu A. Zheng. A systems biology approach to diagnosis and treatments. Science. 2014;346(6216):13-S5.

17. Xu F, Cui W, Kong Q, Tang Z, Dong J. A Real-World Evidence Study for Distribution of Traditional Chinese Medicine Syndrome and Its Elements on Respiratory Disease. Evid Based Complement Alternat Med. 2018;2018:8305892.

18. Kong Q, Jiang R, Li M, Xu F, Zhang Y, Tang Z, et al. A retrospective database analysis of traditional Chinese medicine syndromes in patients with chronic obstructive pulmonary disease and asthma. European Journal of Integrative Medicine. 2019;32.

19. Wang GF, Liu BJ, Cao YX, Du YJ, Zhang HY, Luo QL, et al. Effects of Two Chinese Herbal Formulae for the Treatment of Moderate to Severe Stable Chronic Obstructive Pulmonary Disease: A Multicenter, Double-Blind, Randomized Controlled Trial. Plos One. 2014;9(8).

20. Wei Y, Lyu Y, Li M, Luo Q, Sun J, Liu F, et al. Comparison of effect of granules and herbs of Bu-ShenYi-Qi-Tang on airway inflammation in asthmatic mice. Chin Med J (Engl). 2014;127(22):3957-62.

21. Cui J, Xu F, Tang Z, Wang W, Hu LL, Yan C, et al. Bu-Shen-Yi-Qi formula ameliorates airway remodeling in murine chronic asthma by modulating airway inflammation and oxidative stress in the lung. Biomed Pharmacother. 2019;112:108694.

22. Wei Y, Luo QL, Sun J, Chen MX, Liu F, Dong JC. Bu-Shen-Yi-Qi formulae suppress chronic airway inflammation and regulate Th17/Treg imbalance in the murine ovalbumin asthma model. $J$ Ethnopharmacol. 2015;164:368-77.

23. Luo QL, Nurahmat M, Li MH, Sun J, Chen MX, Liu F, et al. Pharmacological investigation of a HPLC/MS standardized three herbal extracts containing formulae (Bu-Shen-Yi-Qi-Tang) on airway inflammation and hypothalamic-pituitary-adrenal axis activity in asthmatic mice. Phytomedicine. 2014;21(11):1439-50.

24. Sun X, Deng X, Cai W, Li W, Shen Z, Jiang T, et al. Icariin inhibits LPS-induced cell inflammatory response by promoting GRalpha nuclear translocation and upregulating GRalpha expression. Life Sci. 2018;195:33-43.

25. Li L, Sun J, Xu C, Zhang H, Wu J, Liu B, et al. Icariin ameliorates cigarette smoke induced inflammatory responses via suppression of NF-kappaB and modulation of GR in vivo and in vitro. PLoS One. 2014;9(8):e102345.

26. Zhou MQ, Zhuo LY, Cai C. Astragaloside IV Inhibits Cigarette Smoke-Induced Pulmonary Inflammation in Mice. Inflammation. 2018;41(5):1671-80.

27. Xu F, Lin J, Cui W, Kong Q, Li Q, Li L, et al. Scutellaria baicalensis Attenuates Airway Remodeling via $\mathrm{PI}$ KK/Akt/NF-kappaB Pathway in Cigarette Smoke Mediated-COPD Rats Model. Evid Based Complement Alternat Med. 2018;2018:1281420.

28. Wang GF, Mohammadtursun N, Lv YB, Zhang HY, Sun J, Dong JC. Baicalin Exerts Anti-Airway Inflammation and Anti-Remodelling Effects in Severe Stage Rat Model of Chronic Obstructive Pulmonary Disease. Evidence-Based Complementary and Alternative Medicine; 2018. 
29. MH L, AH L, HF L, HK K, TS L. YR K. Paeonol attenuates cigarette smoke-induced lung inflammation by inhibiting ROS-sensitive inflammatory signaling. Mediat Inflamm. 2014;2014:651890.

30. Lin JP, Xu F, Wang GF, Kong LW, Luo QL, Lv YB, et al. Paeoniflorin Attenuated Oxidative Stress in Rat COPD Model Induced by Cigarette Smoke. Evidence-Based Complementary and Alternative Medicine; 2016.

31. McGeachie MJ, Wu AC, Chang HH, Lima JJ, Peters SP, Tantisira KG. Predicting inhaled corticosteroid response in asthma with two associated SNPs. The pharmacogenomics journal. 2013;13(4):306-11.

32. Tantisira KG, Lasky-Su J, Harada M, Murphy A, Litonjua AA, Himes BE, et al. Genomewide association between GLCCI1 and response to glucocorticoid therapy in asthma. $\mathrm{N}$ Engl $\mathrm{J}$ Med. 2011;365(13):1173-83.

33. Maitland-van der Zee AH, Raaijmakers JA. Variation at GLCCI1 and FCER2: one step closer to personalized asthma treatment. Pharmacogenomics. 2012;13(3):243-5.

34. Koster ES, Maitland-van der Zee AH, Tavendale R, Mukhopadhyay S, Vijverberg SJ, Raaijmakers JA, et al. FCER2 T2206C variant associated with chronic symptoms and exacerbations in steroid-treated asthmatic children. Allergy. 2011;66(12):1546-52.

35. Israel E, Chinchilli VM, Ford JG, Boushey HA, Cherniack R, Craig TJ, et al. Use of regularly scheduled albuterol treatment in asthma: genotype-stratified, randomised, placebo-controlled cross-over trial. Lancet. 2004;364(9444):1505-12.

36. Scott SA, Patel M, Martis S, Lubitz SA, van der Zee S, Yoo C, et al. Copy number variation and warfarin dosing: evaluation of CYP2C9, VKORC1, CYP4F2, GGCX and CALU. Pharmacogenomics. 2012;13(3):297-307.

37. O'Meara A, Boukouaci W, Robin M, Xhaard A, Fortier C, Marzais F, et al. GLCCI1 and Glucocorticoid Receptor Genetic Diversity and Response to Glucocorticoid-Based Treatment of Graft-versus-Host Disease. Biology of blood marrow transplantation: journal of the American Society for Blood Marrow Transplantation. 2015;21(7):1246-50.

38. Izuhara Y, Matsumoto H, Kanemitsu Y, Izuhara K, Tohda Y, Horiguchi T, et al. GLCCI1 variant accelerates pulmonary function decline in patients with asthma receiving inhaled corticosteroids. Allergy. 2014;69(5):668-73.

39. Cheng CW, Wu TX, Shang HC, Li YP, Altman DG, Moher D, et al. CONSORT Extension for Chinese Herbal Medicine Formulas 2017: Recommendations, Explanation, and Elaboration. Ann Intern Med. 2017;167(2):112-21.

40. Chan AW, Tetzlaff JM, Altman DG, Laupacis A, Gotzsche PC, Krleza-Jeric K, et al. SPIRIT 2013 statement: defining standard protocol items for clinical trials. Ann Intern Med. 2013;158(3):200-7.

41. Authority TNDR. Administrative Measures for Chinese Medicine Formula Granules (Draft for Comment). 2015.

42. Kong Q, Mo S, Wang W, Tang Z, Wei Y, Du Y, et al. Efficacy and safety of Jia Wei Bushen Yiqi formulas as an adjunct therapy to systemic glucocorticoids on acute exacerbation of COPD: study 
protocol for a randomized, double-blinded, multi-center, placebo-controlled clinical trial. Trials. 2020;21(1):760.

43. Chan A-W, Tetzlaff JM, Gøtzsche PC, Altman DG, Mann H, Berlin JA, et al. SPIRIT 2013 explanation and elaboration: guidance for protocols of clinical trials. BMJ: British Medical Journal. 2013;346:e7586.

\section{Tables}

Table 1

Components and dose of MBYF

\begin{tabular}{|c|c|c|c|c|c|c|}
\hline \multicolumn{2}{|c|}{$\begin{array}{l}\text { Formula } \\
\text { name }\end{array}$} & $\begin{array}{l}\text { Chinese } \\
\text { name }\end{array}$ & $\begin{array}{l}\text { English } \\
\text { name }\end{array}$ & Origin & $\begin{array}{l}\text { Weight } \\
\text { (g)* }\end{array}$ & $\begin{array}{l}\text { Weight } \\
(\%)\end{array}$ \\
\hline \multirow[t]{6}{*}{ MBYF } & \multirow[t]{6}{*}{ BYF } & Huangqi & $\begin{array}{l}\text { Astragali } \\
\text { radix }\end{array}$ & $\begin{array}{l}\text { The dried root of Astragalus } \\
\text { membranaceus (Fisch.). Bge. } \\
\text { Var. Mongholicus (Bge.). Hsiao }\end{array}$ & 1.5 & 14.65 \\
\hline & & Yinyanghuo & $\begin{array}{l}\text { Epimedii } \\
\text { folium }\end{array}$ & $\begin{array}{l}\text { The dried leaf of Epimedium } \\
\text { brevicomu Maxim }\end{array}$ & 0.5 & 4.87 \\
\hline & & Dihuang & $\begin{array}{l}\text { Rehmanniae } \\
\text { Radix }\end{array}$ & $\begin{array}{l}\text { The dried root of Rehmannia } \\
\text { glutinosa Libosch }\end{array}$ & 3 & 29.30 \\
\hline & & Huangqin & $\begin{array}{l}\text { Scutelllariae } \\
\text { radix }\end{array}$ & $\begin{array}{l}\text { The dried root of Scutellaria } \\
\text { baicalensis Georgi }\end{array}$ & 2.24 & 21.87 \\
\hline & & Chishao & $\begin{array}{l}\text { Peaoniae } \\
\text { radix rubra }\end{array}$ & $\begin{array}{l}\text { The dried root of paeonia } \\
\text { lactiflora Pall }\end{array}$ & 3 & 29.30 \\
\hline & & \multicolumn{3}{|l|}{ Total } & 10.24 & $100 \%$ \\
\hline \multicolumn{7}{|c|}{ *The weight of every single herb in each bag of MBYF in granule form } \\
\hline \multicolumn{7}{|c|}{ MBYF: Modified Bushen Yiqi Formulas } \\
\hline
\end{tabular}


Table The hospitals participating in this study

2

\begin{tabular}{lll}
\hline Code & Participating hospitals & Location \\
\hline 01 & Huashan Hospital Affiliated to Fudan University & Eastern China \\
\hline 02 & The First Affiliated Hospital of Nanjing Medical University & Eastern China \\
\hline 03 & $\begin{array}{l}\text { Affiliated Hospital of Traditional Chinese Medicine of Xinjiang Medical } \\
\text { University }\end{array}$ & $\begin{array}{l}\text { Northwestern } \\
\text { China }\end{array}$ \\
\hline 04 & Xinjiang Production and Construction Corps General Hospital & $\begin{array}{l}\text { Northwestern } \\
\text { China }\end{array}$ \\
\hline 05 & The First People's Hospital in Kashgar & Western China \\
\hline 06 & The Second People's Hospital in Kashgar & Western China \\
\hline 07 & Puer Hospital of Traditional Chinese Medicine & $\begin{array}{l}\text { Southwestern } \\
\text { China }\end{array}$ \\
\hline 08 & Yancheng Hospital of Traditional Chinese Medicine & Eastern China \\
\hline 09 & Affiliated Hospital of Nanjing University of Chinese Medicine & Eastern China \\
\hline 10 & Qingpu Traditional Chinese Medicine Hospital attaching & Eastern China \\
\hline
\end{tabular}

Table 3 Diagnostic criteria of TCM syndrome

Primary symptoms

(1) Shortness of breath

(2) Cough

(3) Chest tightness

Secondary symptoms

(1) Tinnitus

(2) Soreness-tired of waist and knee

(3) Spontaneous perspiration囚intolerance to cold, cold hands and feet

(4) Pollakiuria

(5) Lassitude冈fatigue $\llbracket$ disinclination to talk

Due to technical limitations, table 4 is only available as a download in the Supplemental Files section. 
Table 5 variables and method of analysis

\begin{tabular}{|c|c|c|c|}
\hline Outcomes & Hypothesis & Variables & $\begin{array}{l}\text { Analysis } \\
\text { method }\end{array}$ \\
\hline $\begin{array}{l}\text { The frequency and time of } \\
\text { AECOPD }\end{array}$ & \multirow{11}{*}{$\begin{array}{l}\text { MBYF improved } \\
\text { outcomes }\end{array}$} & $\begin{array}{l}\text { Frequency and days } \\
\text { (continuous) }\end{array}$ & ANCOVA \\
\hline The severity of AECOPD & & $\begin{array}{l}\text { Mild, moderate, severe } \\
\text { (categorical) }\end{array}$ & c2 test \\
\hline CAT score & & Score (continuous) & ANCOVA \\
\hline Pulmonary function & & $\begin{array}{l}\text { FEV1\%predicted, FEV1/FEC, } \\
\text { (continuous) }\end{array}$ & ANCOVA \\
\hline Blood gas analysis & & $\mathrm{Pa}(\mathrm{CO} 2), \mathrm{Pa}(\mathrm{O} 2)$ (continuous) & ANCOVA \\
\hline mMRC dyspnea scale & & $0-5$ (categorical) & c2 test \\
\hline 6MWT & & Distance (continuous) & ANCOVA \\
\hline BODE index & & Score (continuous) & ANCOVA \\
\hline $\begin{array}{l}\text { Quantitative scores of TCM } \\
\text { syndrome }\end{array}$ & & Score(continuous) & ANCOVA \\
\hline Inflammation indexes & & $\begin{array}{l}\text { TNF-a,IL-6, IL-8, IL-10, } \\
\text { etc(continuous) }\end{array}$ & ANCOVA \\
\hline HPA axis function & & $\begin{array}{l}\text { ACTH, CRH, CORT } \\
\text { (continuous) }\end{array}$ & $\begin{array}{l}\text { ANCOV } \\
\text { A }\end{array}$ \\
\hline
\end{tabular}

\section{Figures}




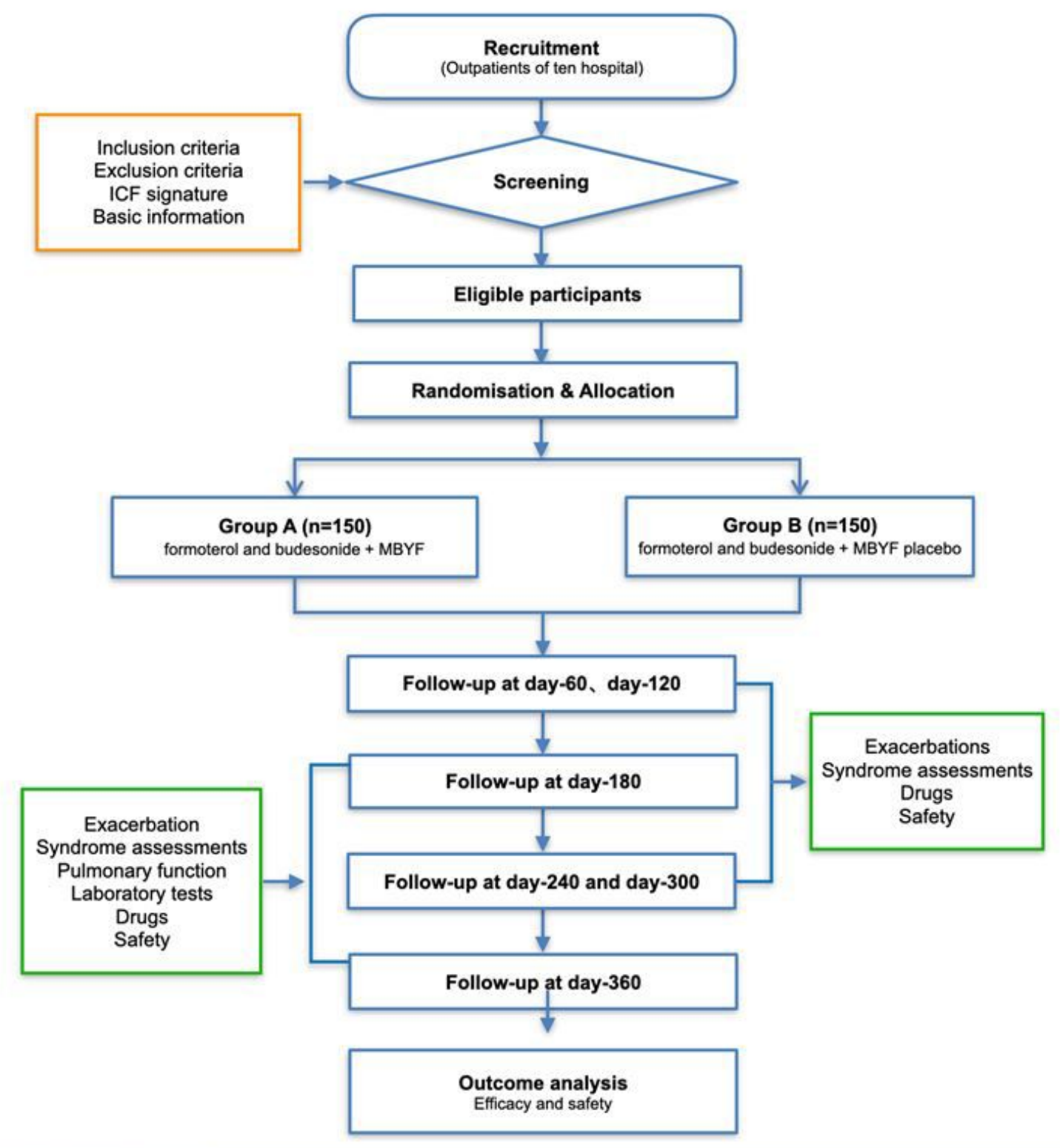

Figure 1

Flow Chart of the study MBYF, modified Bushen Yiqi formulas

\section{Supplementary Files}

This is a list of supplementary files associated with this preprint. Click to download.

- Table4.jpg

- Table4a.jpg

- SPIRITchecklist.doc

- Supplementary.pdf 